\title{
Self-organized criticality in living systems
}

\author{
C. Adami \\ W. K. Kellogg Radiation Laboratory, California Institute of Technology, Pasadena, CA 91125, USA
}

Received 8 December 1994; accepted for publication 28 April 1995

Communicated by C.R. Doering

\begin{abstract}
We suggest that ensembles of self-replicating entities such as biological systems naturally evolve to a self-organized critical state in which fluctuations, as well as waiting times between phase transitions ("epochs"), are distributed according to a $1 / f^{\alpha}$ power law. Such distributions can explain observed frequency distributions in extinction events as well as fractal population structures, and support the punctuated equilibrium picture of evolution. We demonstrate these concepts by analyzing a population of coexisting self-replicating strings (segments of computer code) subject to mutation and survival of the fittest, which constitutes an artificial living system.
\end{abstract}

Self-organized criticality [1] is the term generically applied to systems that are driven to a critical state which is robust to perturbations and whose macroscopic behavior is predictable to the extent that it follows power laws with exponents depending on geometry and spatial structure. In general, the microscopic processes giving rise to the self-organized critical state are dissipative transport processes associated with a threshold, or critical, variable. The paradigm for the self-organized critical state is the sandpile: the critical state is the self-similar and robust pile itself, the distributions of sizes and duration of avalanches (resulting from perturbations) follow distinct power laws, and grains of sand are transported if the local slope of the pile exceeds a critical value, thus restoring the critical state.

It has been suggested [2-6] that biological populations are typically in a self-organized critical state, evidenced for example by a power-law distribution of extinction events. Furthermore, it was observed that population structures gleaned from taxonomic data [7] show a fractal geometry; that is the distribution of subtaxa within taxa is distinctly of the power-law type.

While this is a very appealing idea, especially in view of the robustness of living systems, it has suffered from being somewhat vague, mainly because of the difficulty involved in modeling living systems. Specifically, there is as yet neither a clear identification of the self-organized critical state of life or the agent that causes self-organization, nor a definition of a critical or threshold variable whose disturbance causes the ubiquitous avalanches giving rise to power-law distributions.

Here, we report the observation of self-organized criticality in an artificial living system, the tierra environment $[8,9]$. In this system, strings of machinelanguage-like instructions with the ability to selfreplicate in core memory "live" and co-evolve in an environment subject to random mutation and selection of the fittest. As such, it is not a simulation of life but rather artificial life. Interestingly, the system displays some of the uncanny hallmarks known from 
simple proto-cellular systems, such as growth of the genome and increasing complexity. Most importantly, this artificial environment offers the chance to control the microscopic processes leading to complex behavior, such as replication and mutation. Furthermore, the macroscopic behavior is predictable via the usual methods of statistical mechanics applied to an ensemble of self-replicating entities. As such, this is a unique system to test the hypotheses of self-organized criticality in living systems.

Simple equations $[9,10]$ reveal that in this system the fitness of a string in the population is determined by its replication rate, as measured by executing the string's instructions (its "genome") and counting the number of offspring per unit time. The principle of "survival-of-the-fittest" boils down to a "survival-ofthe-most-populous". Fitness is then a quantity that is genotype specific, i.e., each individual arrangement of instructions in a string translates into a specific replication rate while the survival probability depends on the fitness of the rest of the population. We can thus think of fitness as a highly complicated function on the space of all strings, while the population is characterized by the current average value. Any population, however, is metastable: a successful mutation can create a new "best" genotype (or "master sequence" [11]) with a higher replication rate that disrupts the equilibrium and induces a phase transition to a new "vacuum", defined by the new dominant master sequence and its offspring. Note also that there is no universal "best" string, as the system is in principle infinite and open-ended.

The system of self-replicating strings can be treated in a mean-field approximation that makes it amenable to a statistical description in which the terms "vacuum" and "phase transition" arc precisc informationtheoretic analogues of the respective quantities in conventional statistical systems.

Let $\epsilon_{i}$ stand for the replication rate of genotype $i$, and $\langle\epsilon\rangle$ for its average over the population. In the mean-field approximation, for strings of length $l$ subject to a mutation rate $R$ (we are considering here only external "cosmic-ray" mutations, which have an effect similar to copy-errors), the critical (self-tuned) variable is the growth factor,

$\gamma_{i}=\epsilon_{i}-\langle\epsilon\rangle-R l$.
In the equilibrium situation, the master sequence and its $\epsilon$-degenerate offspring and mutants have $\gamma_{i}=0$, while inferior species have $\gamma_{i}<0$. This prevents exponential growth of the most successful species in the long run. An advantageous mutation, however, can make $\gamma_{i}>0$ for the new master sequence. Such a disturbance causes the information contained in the master sequence to be transmitted throughout the system via the offspring, giving rise to avalanches that are scale-independent. Gradually, all genotypes with a subcritical replication rate will become extinct and be replaced: the system returns to its critical state. Clearly, the normal state of such a population of selfreplicating entities is a superposition of a very large number of metastable states, with transitions between them induced by mutation and copy-errors. It undergoes spontaneous phase transitions if a mutation creates a genotype with $\gamma_{i}>0$, ushering in a new "epoch" of domination by a new species. In fact, in such a system there is no scale that would set the average time between avalanches, nor is there a scale setting the size of the avalanche. The latter is determined by the amount of information gained by the new master sequence. In a system with an infinite "supply" of information (complex fitness landscape), we thus expect both distributions to be given by power laws.

To test this hypothesis, we have analyzed the evolution of an ensemble of strings subject to Poissonrandom mutations at a rate of $R=0.5 \times 10^{-8}$ mutations per site per unit time (the unit of time is the execution of one instruction). The strings are segments of computer code of a specially developed instruction set with only 32 instructions running on a virtual computer. A mutated instruction most likely will cause the program to "break", yet occasionally may improve it. The strings live in a strip of memory with a total number of 131072 sites that can represent one instruction each, with periodic boundary condition (i.e., the strip wraps on itself). It is typically inhabited by $600-$ 1400 strings of length $60-150$, all offspring of a single (handwritten) progenitor that is able to self-replicate and used to "inoculate" the strip. For purposes of reproducibility, we used the original "ancestor" written by Ray [8], who created the tierra system. As described elsewhere [9], this ancestor is well-suited for evolutionary experiments due to the amount of redundancy in its code. The fitness landscape that this population explores is determined $b_{y}$ all possible ways to 


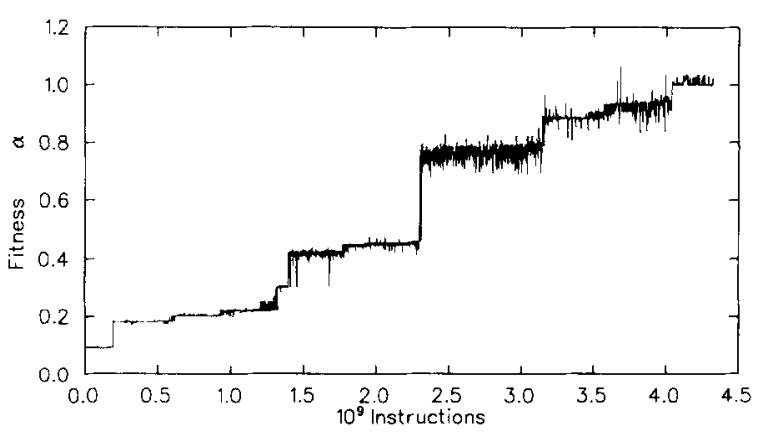

Fig. 1. Fitness curve for a typical run. The fitness parameter $\alpha$ of the most successful (i.e., most populous) genotype is plotted as a function of time, measured every million instructions for a mutation rate $R=0.5 \times 10^{-8}$.

reduce the time to gestate a single offspring (the gestation time) and the opportunity to trigger bonus CPU time by developing the "genetic code" necessary to perform certain user-specified tasks (see Ref. [9] for details on this environment). In other words, we provide an environment containing information that the strings can discover (through adaptive mutation) and exploit.

The population adapts to this environment through discontinuous jumps, as evidenced in Fig. 1. There, we have plotted the "fitness-of-the-best" $\alpha$ versus total number of instructions executed (i.e., time elapsed) every million instructions for a typical run. For technical reasons, the measured quantity $\alpha$ is the replication rate $\epsilon$ multiplied by the total number of instructions allocated to the strings in one "sweep" through the population, with $0 \leqslant \alpha \leqslant 1$. The latter bound is imposed only to maintain parallelism: in order to emulate parallel coexistence, each string is allocated a certain slice of CPU time and executed serially (see, e.g., Ref. [8] for details). The visible noise in Fig. 1 is mainly due to mutations and finite-size effects. This noise, of course, drives the fitness jumps that adapt the population to the environment.

We have plotted in Fig. 2 the power spectral density of a typical fitness history, which reveals a clear power-law distribution with $P(f) \sim f^{-\beta}$ and $\beta=$ $2.0 \pm 0.05$. Scaling exponents from other runs are compatible within the error bars quoted.

Fluctuations distributed according to a power law are the telltale sign of a self-organized critical state [1]. However, since there certainly are systems (such

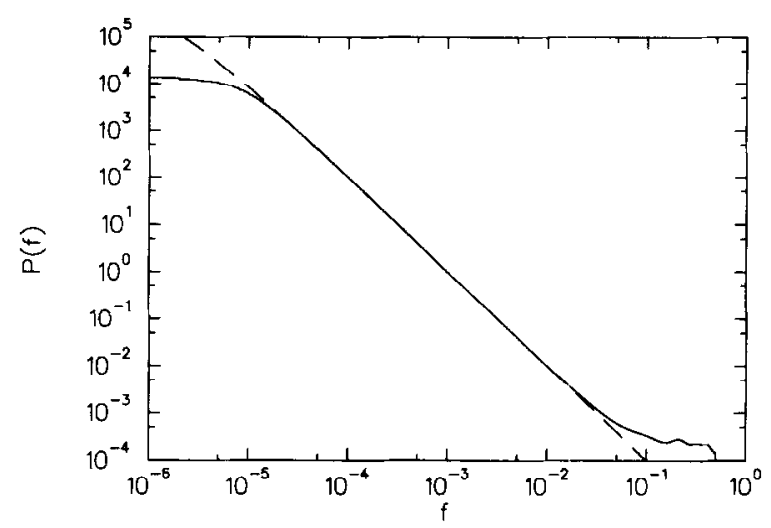

Fig. 2. Power spectrum $P(f)$ of a typical fitness curve $\alpha(t)$ (Fig. 1). The dashed line is a fit to $P(f) \sim f^{-\beta}$ with $\beta=2.0 \pm 0.05$.

as random walks) with identical power-law spectra which are not self-organized, we have also measured the distribution of waiting times between phase transitions, or length of epochs, in 50 runs under identical conditions (save the random number seed), resulting in 512 measured waiting times.

To obtain the waiting times, we determined that a phase transition occurred if the fitness jumps discontinually to a new level with a fitness increase of a minimum of $7.5 \%$. As the resulting plot strongly suggests that the fitness curves are fractal, this condition cannot change the power law. Rather, fitness curves like the one in Fig. 1 are expected to look similar at all scales (devil's staircase [12]). Clearly, as we cannot measure waiting times ${ }^{1} t \geqslant 500$ with good statistical accuracy due to the finite lengths of our runs, the distribution function shows finite-size effects that we model with a cutoff parameter $T$,

$N(\tau) \sim \tau^{-\alpha} \mathrm{e}^{-\tau / T}$.

Statistically more reliable is the integrated distribution function

$$
M(\tau)=\frac{1}{\tau} \int_{\tau}^{\infty} N(t) \mathrm{d} t \sim \frac{1}{\tau} \Gamma(1-\alpha, \tau / T),
$$

\footnotetext{
${ }^{\mathrm{I}}$ Waiting times are measured in units of millions of instructions executed. Most runs where stopped after between 500 and 2000 million instructions where executed.
} 


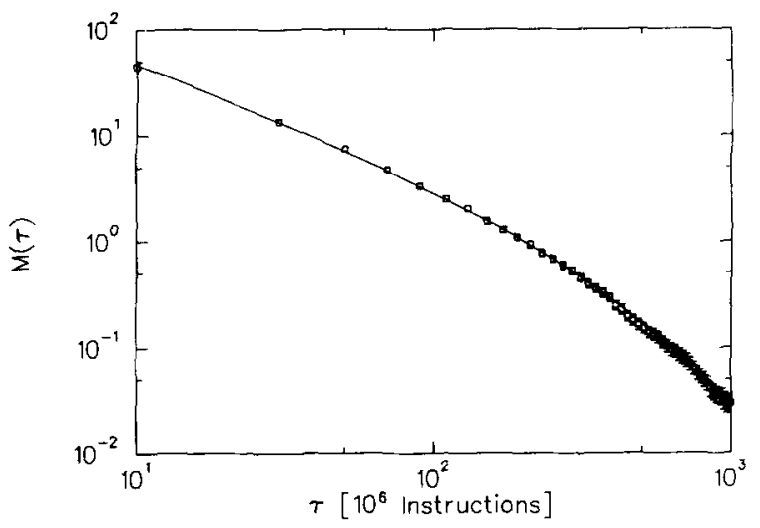

Fig. 3. Integrated distribution of times between phase transitions $\tau$ (length of epoch). The solid line is a fit to the incomplete gamma function with $\alpha=0.6 \pm 0.1$ and a cutoff parameter $T=540 \pm 40$ modeling finite-size effects.

where $\Gamma(1-\alpha, \tau / T)$ is an incomplete gamma function, which is distributed with the same power-law exponent as $N(\tau)$. Indeed the resulting fit (shown in Fig. 3) yields $\alpha=0.6 \pm 0.1$ and $T=540 \pm 40$ which agrees with the fit obtained from $N(\tau)$ within error bars, but is more accurate. While we expect the coefficient $\alpha$ to be universal, the cutoff depends on the average length of runs.

A power-law distribution in waiting times has a number of significant consequences. First, the distribution of waiting times between events of a certain size in a random walk model is exponential rather than of the power-law type, as is the distribution in all percolation-type systems not tuned to the critical variable. It appears thus that temporal correlations are very sensitive to the level of self-organization and the criticality of the model. Further, a power-law distribution of epochs in a model of evolution may solve a puzzling problem in biology, namely why there is a fractal structure in taxonomic systems [7]. Indeed, if the number of subspecies that a species generates is proportional to the time it dominates the population, a power-law distribution of waiting times implies a power-law distribution in the number of species $N$ with $n$ subspecies, as measured by Burlando [7].

We have suggested that the normal state of an ensemble of self-replicating entities is self-organized criticality, the agent of self-organization being information. We identified the growth factor $\gamma_{i}$ as the critical variable and described avalanches of "invention" that drive the adaptation of the population. We tested these hypotheses in the artificial life system tierra and found power-law distributions in the power spectrum of fitness fluctuations, as well as in the distribution of waiting times. Self-organized criticality in living systems has wide-ranging consequences for theories of evolution. On the one hand, gradualism is incompatible with criticality, and a punctuated equilibrium picture is favoured (see, e.g., Ref. [13]). On the other hand, the fractal nature of the fitness history (Fig. 1) would account for fitness improvements on all scales driven only by microscopic mutations. Furthermore, SOC in living systems may explain the fractal structure in the taxonomic system as measured recently.

Note added. After completion of this manuscript, we became aware of Ref. [14], in which conclusions similar to ours are drawn from a simple evolutionary model.

This work was supported in part by the National Science Foundation Grant Nos. PHY91-15574 and PHY94-12818, as well as a Caltech Division Fellowship.

\section{References}

(1) P. Bak, C. Tang and K. Wiesenfeld, Phys. Rev. Lett. 59 (1987) 381; Phys. Rev. A 38 (1988) 364.

[2] M. Eigen, Adv. Chem. Phys. 33 (1978) 211.

[3] D.M. Raup. Science 231 (1986) 1528.

14] S.A. Kauffman and S. Johnsen, J. Theor. Biol. 149 (1991) 467.

[5] S.A. Kauffman, The origins of order (Oxford Univ. Press, Oxford, 1993)

[6] P. Bak, Physica A 191 (1992) 41.

17] B. Burlando. J. Theor. Biol. I46 (1990) 99; 163 (1993) 161.

18| T.S. Ray, in: Santa Fe Institute Studies in the Sciences of Complexity, Vol. 10. Artificial life II: Proc. Interdisciplinary Workshop on the Synthesis and simulation of living systems, eds. C.G. Langton et al. (Addison-Wesley, Reading, MA, 1992) p. 371.

[9] C. Adami, Physica D 80 (1995) 154.

[10] C. Adami. Artif. Life 1 (1994) 429.

[ 11 ] M. Eigen, J. McCaskill and P. Schuster, Adv. Chem. Phys. 75 (1989) 149.

[12] B. Mandelbrot, The fractal geometry of nature (Freeman, San Francisco, 1977).

[13] S.J. Gould and N. Eldredge, Nature 366 (1993) 223.

[14] P. Bak and K. Sneppen, Phys. Rev. Lett. 71 (1993) 4083. 